\title{
Amniotic Fluid L-Type Fatty Acid-Binding Protein in Predicting Fetal Condition
}

\author{
Daisuke Katsura, ${ }^{1}$ Shunichiro Tsuji, ${ }^{1}$ Kaori Hayashi, ${ }^{1}$ Shinsuke Tokoro, ${ }^{1}$ \\ Rika Zen, ${ }^{1}$ Takako Hoshiyama, ${ }^{1}$ Fuminori Kimura, ${ }^{1}$ Nobuyuki Kita ${ }^{1}$ and \\ Takashi Murakami ${ }^{1}$ \\ ${ }^{1}$ Department of Obstetrics and Gynecology, Shiga University of Medical Science Hospital, Otsu, Shiga, Japan
}

\begin{abstract}
Ultrasonography and fetal heart rate monitoring are subjective assessments of fetal condition, which warrants the need for objective markers to predict fetal condition. Urinary L-type fatty acid-binding protein (L-FABP) levels correlate with hypoperfusion. Elevated amniotic fluid L-FABP levels may represent fetal tissue hypoperfusion since the amniotic fluid contains fetal urine. In this study, we aimed to analyze the effectiveness of amniotic fluid L-FABP as a predictor of fetal condition. We classified singleton pregnancies into groups based on fetal growth restriction (FGR) with and without fetal blood flow abnormalities (FGR and healthy-FGR groups, respectively) and the non-FGR group (control group). We collected amniotic fluid at the time of vaginal delivery, cesarean section and amniocentesis, and compared the patient characteristics, clinical outcomes and amniotic fluid levels of L-FABP between the groups. We analyzed 153 singleton pregnancies and 186 amniotic fluid samples (FGR group, 6 (3.9\%) pregnancies and 23 $(12.4 \%)$ samples; healthy-FGR group, 15 (9.8\%) pregnancies and $20(10.7 \%)$ samples; control group, 132 (86.3\%) pregnancies and $143(76.9 \%)$ samples). The amniotic fluid L-FABP level was significantly higher in the FGR group compared to that in the healthy-FGR and control groups. Multivariate analysis revealed that the amniotic fluid L-FABP level was not affected by fetal body weight. Additionally, the amniotic fluid L-FABP levels increased significantly in cases with fetal blood flow abnormalities or early gestational age. Therefore, amniotic fluid L-FABP level may be an objective and accurate predictive marker of fetal condition.
\end{abstract}

Keywords: amniotic fluid; fetal blood flow abnormality; fetal condition; fetal growth restriction; L-type fatty acidbinding protein

Tohoku J. Exp. Med., 2021 August, 254 (4), 267-273.

\section{Introduction}

The fetal condition during pregnancy is assessed using ultrasonography and fetal heart rate monitoring in the form of the biophysical profile score (Manning 1999). In the event of fetal growth restriction (FGR), the timing of delivery is determined with the goal of preventing fetal death by predicting fetal deterioration according to gestational age, determining the degree of fetal blood flow abnormalities, and monitoring the fetal heart rate (The GRIT Study Group 1996; Lees et al. 2015). However, it is important not only to prevent fetal death but also to consider the short-and long-term prognoses after birth. The relationship between absent or reversed end-diastolic velocity in the umbilical artery, and chronic lung disease, retinopathy of prematurity, impaired intestinal motility, mental retardation, motor impairment, hearing loss and cerebral palsy has been reported. Further, the relationship between abnormal ductus venosus velocity and abnormal motor development has also been reported (Vossbeck et al. 2001; Baschat et al. 2009). Although fetal blood flow abnormalities might be associated with short- and long-term prognoses (Vossbeck et al. 2001; Baschat et al. 2009), it has been suggested that the intrauterine environment of the fetus is also associated with short- and long-term prognoses, such as developmental origins of health and disease (Gluckman et al. 2005, 2007; Baschat 2011). Fetal Doppler velocity and fetal heart rate monitoring may be useful in determining the current

Received April 28, 2021; revised and accepted June 5, 2021. Published online August 21, 2021; doi: 10.1620/tjem.254.267.

Correspondence: Daisuke Katsura, M.D., Department of Obstetrics and Gynecology, Shiga University of Medical Science Hospital, Seta Tsukinowa-cho, Otsu, Shiga 520-2192, Japan.

e-mail: katsuo14@belle.shiga-med.ac.jp

(C)2021 Tohoku University Medical Press. This is an open-access article distributed under the terms of the Creative Commons Attribution-NonCommercial-NoDerivatives 4.0 International License (CC-BY-NC-ND 4.0). Anyone may download, reuse, copy, reprint, or distribute the article without modifications or adaptations for non-profit purposes if they cite the original authors and source properly.

https://creativecommons.org/licenses/by-nc-nd/4.0/ 
fetal condition and predicting fetal deterioration; however, predicting the intrauterine environment in which the fetus is placed is difficult. The degree and course of fetal Doppler velocity and fetal heart rate monitoring might be useful in predicting the intrauterine environment; however, it is difficult to adequately monitor them continuously. Furthermore, fetal Doppler velocity and fetal heart rate monitoring are subjective assessments. Thus, objective and accurate markers to predict fetal condition and the intrauterine environment are needed. These might lead to an improved ability to time a delivery, considering not only fetal death but also short- and long-term prognoses.

Urinary L-type fatty acid-binding protein (L-FABP) may be useful in detecting acute kidney injury caused by septic shock, and L-FABP levels correlate with hypoperfusion or oxidative stress (Asada et al. 2016). Elevated amniotic fluid L-FABP levels may represent fetal tissue hypoperfusion since the amniotic fluid contains fetal urine. Thus, this biomarker may be a useful factor in the evaluation of fetal condition and the intrauterine environment.

In this study, we aimed to evaluate the utility of amniotic fluid L-FABP levels as a predictor of fetal condition and the intrauterine environment.

\section{Materials and Methods}

\section{Patients}

We included singleton pregnancies that were managed and delivered at the Department of Obstetrics and Gynecology, Shiga University of Medical Science Hospital, Shiga, Japan, between August 2020 and March 2021. Pregnancies with chromosomal and genetic abnormalities and major structural anomalies were excluded. The cases were classified according to FGR with fetal blood flow abnormalities such as umbilical artery and ductus venosus pulsatility index $>95^{\text {th }}$ percentile, umbilical artery with absent or reversed end-diastolic velocity, and ductus venosus with absent or reversed A-wave (FGR group); FGR without fetal blood flow abnormalities (healthy-FGR group); and no FGR (control group). FGR was diagnosed based on the Japanese Society of Ultrasound in Medicine standards $(<-1.5$ standard deviation) (Shinozuka 2002). The timing of delivery was usually decided according to the obstetric indications, such as a non-reassuring fetal status on fetal heart rate monitoring, and the maternal condition, including severe hypertensive disorders of pregnancy.

The timing of delivery for fetuses with preterm FGR remains controversial. Therefore, at our institution, the following indications were followed for delivery of these fetuses: ductus venosus with absent or reversed A-wave in fetuses with gestational age $>23$ weeks and estimated weight $>300 \mathrm{~g}$; reversed end-diastolic velocity in the umbilical artery in fetuses with gestational age > 32 weeks; umbilical artery with absent end-diastolic velocity in fetuses with gestational age $>34$ weeks. All procedures were performed in accordance with the Declaration of Helsinki. This study protocol was approved by the Institutional
Review Board of the Shiga University of Medical Science Hospital. Informed consent was obtained from all patients.

\section{Data measurement}

To measure the amniotic fluid L-FABP levels, amniotic fluid samples were collected transvaginally following the rupture of membranes in vaginal delivery, or using an 18 -gauge needle to puncture the membrane before rupture in cesarean delivery. Additionally, amniotic fluid samples were collected during amniocentesis for chromosomal examination, amnioreduction for polyhydramnios, and amnioinfusion for relieving umbilical cord compression due to oligohydramnios in cases with fetal blood flow abnormalities (Takahashi et al. 2014; Iwagaki et al. 2019; Katsura et al. 2019). The samples were refrigerated at $-4^{\circ} \mathrm{C}$ until the analysis was performed. The L-FABP levels were measured using the two-step sandwich Chemiluminescent Enzyme Immunoassay kit following the manufacturer's protocol (SRL, Tokyo, Japan). The influence of variation in the urine flow rate should be considered for adequate evaluation of urinary biomarkers. Creatinine correction reportedly has a higher diagnostic power for acute kidney injury than urine flow rate correction (Tonomura et al. 2011). The creatinine-corrected value was adopted for the amniotic fluid L-FABP levels since it is difficult to evaluate the amount of amniotic fluid and fetal urine flow rate accurately.

All pulse Doppler measurements were recorded by experienced operators using a wall motion filter of $60 \mathrm{~Hz}$ and a gate-size fitting within the blood vessels by evaluating five or more consecutive waveforms. The umbilical artery was measured from the abdominal insertion of the umbilical cord to the fetal pelvis to avoid the effect of abnormal resistance from a superficial placental anastomosis. If the umbilical artery had an absent or reversed enddiastolic velocity or if the ductus venosus had an absent or reversed A-wave, it was confirmed by lowering the setting further.

We collected data on maternal characteristics and clinical outcomes, including the maternal age, parity, body mass index, gestational age at delivery, mode of delivery, birth weight, and umbilical artery $\mathrm{pH}$. We also recorded neonatal outcomes, including hospitalization in the neonatal intensive care unit (NICU), need for respiratory support such as nasal directional positive airway pressure and conventional mechanical ventilation, chronic lung disease, periventricular leukomalacia, necrotizing enterocolitis, intraventricular hemorrhage, retinopathy of prematurity, and neonatal death.

\section{Statistical analyses}

We used Fisher's exact probability test, KruskalWallis test, and one-way analysis of variance (ANOVA) to compare the data among the FGR, healthy-FGR, and control groups. In the Kruskal-Wallis test and one-way ANOVA, significant values for the groups were adjusted 
using the Bonferroni correction. A p-value $<0.05$ was considered statistically significant. The correlation between the amniotic fluid L-FABP levels and the ductus venosus pulsatility index was assessed using Pearson's correlation coefficient. Statistical analyses were performed using Easy R (EZR; R Foundation for Statistical Computing, Vienna, Austria) (Kanda 2013).

\section{Results}

This study included 180 singleton pregnancies. We excluded 5 pregnancies with chromosomal or genetic abnormalities and 11 with major structural anomalies. Consequently, 163 single pregnancies and 196 amniotic fluid samples were included. We performed univariate analysis for the amniotic fluid collection method, presence or absence of blood contamination, onset of labor, and turbidity to investigate the factors that affect the L-FABP levels. The turbidity and blood contamination significantly affected the L-FABP levels $(p<0.001$ and $p=0.032$, respectively), whereas the amniotic fluid collection method, blood contamination, and onset of labor did not influence the L-FABP levels $(\mathrm{p}=0.354, \mathrm{p}=0.128$, and $\mathrm{p}=0.615$, respectively). In the multivariate analyses for turbidity and blood contamination, only turbidity affected the L-FABP levels significantly $(p<0.001)$. Therefore, we excluded 10 cases with turbidity. Finally, 153 singleton pregnancies and
186 amniotic fluid samples were analyzed. Six (3.9\%) pregnancies had FGR with fetal blood flow abnormalities (FGR group) and constituted $23(12.4 \%)$ amniotic fluid samples; 15 (9.8\%) patients had FGR without fetal blood flow abnormalities (healthy-FGR group) and constituted 20 (10.7\%) amniotic fluid samples; $132(86.3 \%)$ patients did not have FGR (control group) and constituted 143 (76.9\%) amniotic fluid samples. The demographic data, characteristics, and clinical outcomes are summarized in Table 1, which highlights significant differences in the gestational age at amniotic fluid collection and delivery, birth weight and prevalence of NICU admission, respiratory support, and neonatal death. There were no cases of chronic lung disease, periventricular leukomalacia, or necrotizing enterocolitis.

The FGR group included three cases of umbilical hyper-coiled cords, one case of umbilical hyper-coiled cord and true knot of the umbilical cord, one case of multiple placental infarctions, and an unknown cause in one case.

Significant differences were observed in the amniotic fluid L-FABP levels among the groups $(p<0.001)$, and the amniotic fluid L-FABP level was significantly higher in the FGR group, compared to the healthy-FGR and control groups (Table 2). We performed multivariate analyses using the multiple regression model for FGR with and without fetal blood flow abnormalities, gestational age at amni-

Table 1. Patient characteristics and clinical outcomes of patients in the control, healthy- fetal growth restriction (FGR), and FGR groups.

\begin{tabular}{|c|c|c|c|c|c|c|}
\hline \multirow{2}{*}{ Characteristics } & \multirow{2}{*}{$\begin{array}{l}\text { 1. Control } \\
(\mathrm{n}=132)\end{array}$} & \multirow{2}{*}{$\begin{array}{l}\text { 2. Healthy-FGR } \\
\quad(\mathrm{n}=15)\end{array}$} & \multirow{2}{*}{$\begin{array}{l}\text { 3. FGR } \\
(\mathrm{n}=6)\end{array}$} & \multicolumn{3}{|c|}{ p-Value } \\
\hline & & & & 1 vs. 2 & 2 vs. 3 & 1 vs. 3 \\
\hline Number of amniotic fluid samples & 143 & 20 & 23 & N/A & $\mathrm{N} / \mathrm{A}$ & N/A \\
\hline Age (years) ${ }^{\mathrm{a}}$ & $33.5[18-47]$ & $31.0[22-43]$ & $32.0[21-41]$ & 0.640 & 1 & 0.850 \\
\hline Primipara $^{\mathrm{b}}$ & $61(46.2 \%)$ & $10(66.7 \%)$ & $2(33.3 \%)$ & 0.175 & 0.331 & 0.688 \\
\hline $\mathrm{IVF}^{\mathrm{b}}$ & $24(18.2 \%)$ & $6(40 \%)$ & 0 & 0.082 & 0.123 & 0.590 \\
\hline BMI $\left(\mathrm{kg} / \mathrm{m}^{2}\right)^{\mathrm{a}}$ & $21.2[14.2-45.1]$ & $23.0[16.1-43.9]$ & $25.3[18.3-27.7]$ & 1 & 0.350 & 0.350 \\
\hline $\mathrm{GDM}^{\mathrm{b}}$ & $11(8.3 \%)$ & 0 & 0 & 0.604 & 1 & 1 \\
\hline $\mathrm{HDP}^{\mathrm{b}}$ & $14(10.6 \%)$ & $3(20 \%)$ & 0 & 0.384 & 0.526 & 1 \\
\hline GA at amniotic fluid collection (weeks) & $38.1[27.0-41.3]$ & $35.3[30.6-40.9]$ & $28.4[20-38.2]$ & 0.17 & $<0.001$ & $<0.001$ \\
\hline GA at delivery (weeks) ${ }^{\mathrm{a}}$ & $38.1[28.3-41.3]$ & $38.0[33.7-40.9]$ & $31.1[26.9-38.3]$ & 0.435 & 0.133 & 0.014 \\
\hline $\mathrm{CS}^{\mathrm{b}}$ & $63(47.7 \%)$ & $9(60 \%)$ & $5(83.3 \%)$ & 0.422 & 0.613 & 0.113 \\
\hline Birth weight $(\mathrm{g})^{\mathrm{a}}$ & $2,918[1,032-3,786]$ & $2,076[1,206-2,688]$ & $892[307-2,104]$ & $<0.001$ & 0.098 & $<0.001$ \\
\hline Umbilical artery $\mathrm{pH}^{\mathrm{a}}$ & $7.296[7.109-7.412]$ & $7.266[7.129-7.353]$ & $7.282[7.234-7.348]$ & 0.830 & 1 & 1 \\
\hline NICU admission ${ }^{\mathrm{b}}$ & $38(28.8 \%)$ & $11(73.3 \%)$ & $6(100 \%)$ & 0.001 & 0.003 & $<0.001$ \\
\hline Respiratory support ${ }^{\mathrm{b}}$ & $16(12.1 \%)$ & $4(26.7 \%)$ & $6(100 \%)$ & 0.125 & 0.003 & $<0.001$ \\
\hline $\mathrm{IVH}^{\mathrm{b}}$ & $1(0.8 \%)$ & 0 & 0 & 1 & 1 & 1 \\
\hline $\mathrm{ROP}^{\mathrm{b}}$ & $1(0.8 \%)$ & 0 & $1(16.7 \%)$ & 1 & 0.286 & 0.085 \\
\hline Neonatal death ${ }^{\mathrm{b}}$ & 0 & 0 & $1(16.7 \%)$ & 1 & 0.039 & 0.043 \\
\hline
\end{tabular}

Data is expressed as median [range] or $\mathrm{n}(\%)$.

aKruskal-Wallis test was used for between-group comparisons, and significant values were adjusted using Bonferroni correction for the groups.

'Fisher's exact test was used for between-group comparisons.

FGR, fetal growth restriction; IVF, in vitro fertilization; BMI, body mass index; GDM, gestational diabetes; HDP, hypertensive disorders of pregnancy; GA, gestational age; CS, cesarean section; NICU, neonatal intensive care unit; IVH, intraventricular hemorrhage; ROP, retinopathy of prematurity; N/A, not applicable. 
otic fluid collection, and birth weight to investigate the factors that affect the amniotic fluid L-FABP level. We observed that fetal blood flow abnormalities and gestational age at amniotic fluid collection significantly affected the amniotic fluid L-FABP levels $(\mathrm{p}<0.001$ and $\mathrm{p}=0.029$, respectively), whereas FGR and birth weight did not influence the L-FABP levels $(p=0.329$, and 0.268 , respectively).

The relationship between the amniotic fluid L-FABP levels and fetal blood flow abnormalities are summarized in Table 3. The analysis was performed after the exclusion of 2 cases with ductus venosus with absent A-wave, and one case with umbilical artery with reversed end-diastolic velocity since they comprised a small sample size. The amniotic fluid L-FABP level was significantly higher in cases with ductus venosus with a reversed A-wave than that in cases with ductus venosus pulsatility index $>95^{\text {th }}$ percentile. No statistically significant differences were observed in other fetal blood flow abnormalities (Table 3). The Pearson's correlation coefficient for L-FABP and ductus venosus pulsatility index inspection was $\mathrm{r}=-0.189(\mathrm{p}=$ 0.627 ), and no correlation was observed between them.

In the FGR group, the changes in L-FABP levels during pregnancy until delivery were confirmed in three cases. In case 1, the L-FABP levels gradually increased (Fig. 1A), and an emergency cesarean section was performed at 30 weeks and 6 days of gestation due to non-reassuring fetal status on fetal heart rate monitoring. In case 2, L-FABP levels temporarily increased and then decreased (Fig. 1B), and an elective cesarean section was performed at 34 weeks and 1 day of gestation due to oligohydramnios and breech presentation. In case 3, L-FABP levels remained high flat but rapidly increased (Fig. 1C), and an emergency cesarean section was performed at 26 weeks and 6 days of gestation after ductus venosus with reversed A-wave and fetal estimated weight $>300 \mathrm{~g}$ were confirmed on ultrasonography. A male infant weighing $307 \mathrm{~g}$ was delivered with an umbilical artery $\mathrm{pH}$ of 7.245. Case 3 resulted in neonatal death.

Neonatal creatinine was measured in 50 cases, and the median creatinine level was $0.525 \mathrm{mg} / \mathrm{L}$ (range, 0.39-0.88). The Pearson's correlation coefficient for L-FABP and neonatal creatinine measurement was $r=0.005(p=0.968)$, and no correlation was observed between them.

\section{Discussion}

The intrauterine environment to which a fetus is exposed to during pregnancy is believed to reflect the shortand long-term prognoses (Gluckman et al. 2005, 2007; Baschat 2011). Fetal Doppler velocity and fetal heart rate monitoring express the fetal condition and can predict fetal deterioration (The GRIT Study Group 1996; Manning 1999; Lees et al. 2015); their courses might predict the intrauterine environment as well. However, they are subjective assessments, and it is difficult to adequately monitor them continuously. Objective and accurate markers that can comprehensively evaluate only the fetal condition are desired. The findings of this study demonstrated that the amniotic fluid L-FABP levels are not affected by fetal body

Table 2. Amniotic fluid L-FABP levels of patients in the control, healthy-FGR, and FGR groups.

\begin{tabular}{|c|c|c|c|}
\hline & Control $(n=143)$ & Healthy-FGR $(\mathrm{n}=20)$ & FGR $(n=23)$ \\
\hline L-FABP $(\mu \mathrm{g} / \mathrm{gCr})^{\mathrm{a}}$ & $197.6 \pm 251.0$ & $258.5 \pm 206.1$ & $3,220.8 \pm 2,190.7$ \\
\hline \multicolumn{4}{|l|}{ p-Value } \\
\hline vs. control & & 1 & $\mathrm{P}<0.001$ \\
\hline vs. Healthy-FGR & & & $\mathrm{P}<0.001$ \\
\hline
\end{tabular}

Data are expressed as mean \pm standard deviation.

a One-way analysis of variance was used for between-group comparisons, and significant values were adjusted using the Bonferroni correction for the group.

L-FABP, L-type fatty acid-binding protein; FGR, fetal growth restriction.

Table 3. Relationship between amniotic fluid L-FABP levels and fetal blood flow abnormalities in patients of the FGR groups.

\begin{tabular}{|c|c|c|c|c|c|}
\hline & $\operatorname{DVR}(\mathrm{n}=7)$ & $\operatorname{DVA}(\mathrm{n}=2)$ & $\operatorname{UAR}(\mathrm{n}=1)$ & $\operatorname{UAA}(\mathrm{n}=4)$ & DVPI $>95^{\text {th }}(\mathrm{n}=10)$ \\
\hline L-FABP $(\mu \mathrm{g} / \mathrm{gCr})^{\mathrm{a}}$ & $5,010[1,290-9,490]$ & $1,600[1,250-1,950]$ & 2,020 & $1,445[1,190-2,300]$ & $2,915[1,100-4,260]$ \\
\hline \multicolumn{6}{|l|}{ p-Value } \\
\hline vs. DVR & & & & 0.095 & 0.005 \\
\hline vs. UAA & & & & & 1 \\
\hline
\end{tabular}

Data is expressed as median [range].

aKruskal-Wallis test was used for between-group comparisons, and significant values were adjusted using the Bonferroni correction for the groups.

L-FABP, L-type fatty acid-binding protein; FGR, fetal growth restriction; DVR, ductus venosus with reversed A-wave; DVA, ductus venosus with absent A-wave; UAR, umbilical artery with reversed end-diastolic velocity; UAA, umbilical artery with absent end-diastolic velocity; DVPI $>95^{\text {th }}$, ductus venosus pulsatility index $>95^{\text {th }}$ percentile. 
weight and that they increase significantly in cases with fetal blood flow abnormalities or early gestational age. Amniotic fluid L-FABP level is suggested to directly and comprehensively reflect the fetal condition and is an objective and accurate marker, compared with fetal Doppler velocity and fetal heart rate monitoring.

The FABP family comprises nine subtypes according to organ-specific expression, some of which are closely linked to ischemic tissue conditions (Yamamoto et al. 2007). FABP serves as a target for highly cytotoxic aldehydes that are inevitably generated in lipid peroxidation reactions in response to oxidative stress, thereby reducing lipid peroxidative stress (Yamamoto et al. 2007; Derikx et al. 2007). L-FABP is expressed in the liver and the proximal epithelial tubules (Yamamoto et al. 2007; Asada et al. 2016); moreover, in vitro expression of L-FABP has been reported to reduce oxidative stress during hypoxia-reoxygenation (Wang et al. 2005). In response to oxidative stress, the L-FABP binds with and transfers cytotoxic aldehydes produced during lipid peroxidation to the urinary space and sheds it into the urine (Bennaars-Eiden et al. 2002). In a study using the mouse model, expression of renal L-FABP was suggested to protect the kidney tissue from renal ischemic stress (Yamamoto et al. 2007). Therefore, elevated urinary L-FABP levels have been shown to be effective early indicators of ischemic conditions (Yamamoto et al. 2007). This implies that elevated amniotic fluid L-FABP levels might reflect fetal hypoxia since the amniotic fluid contains fetal urine. In this study, there was a significant relationship between L-FABP levels and FGR with fetal blood flow abnormalities; however, there was no relationship between the L-FABP levels and healthy-FGR. Although birth weight was significantly lower and the prevalence of NICU admission was significantly higher in the healthy-FGR group than those in the control group, there was no significant difference in the prevalence of short-term prognosis, including respiratory support, between the healthy-FGR and control groups. Additionally, fetuses with an estimated fetal weight $<3^{\text {rd }}$ percentile and no blood flow abnormalities are called constitutionally small for gestational age, and reportedly have virtually normal perinatal outcomes (Figueras and Gratacos 2014). Therefore, we considered this to be the reason for no increase in the amniotic fluid L-FABP levels in the healthy-FGR group. Additionally, there was a significant relationship between the L-FABP levels and cases with early gestational age. Although the mechanism by which gestational age influences the L-FABP levels remains unclear, elevated amniotic fluid L-FABP levels in cases with early gestational age might be speculated to reflect the immaturity of the fetus. This requires further research in future studies.

Statistically significant differences were not confirmed in the amniotic fluid L-FABP levels between the types of fetal blood flow abnormalities, except between cases with ductus venosus with a reversed A-wave and those with ductus venosus pulsatility index $>95^{\text {th }}$ percentile. The reason might have been due to the small number of amniotic fluid
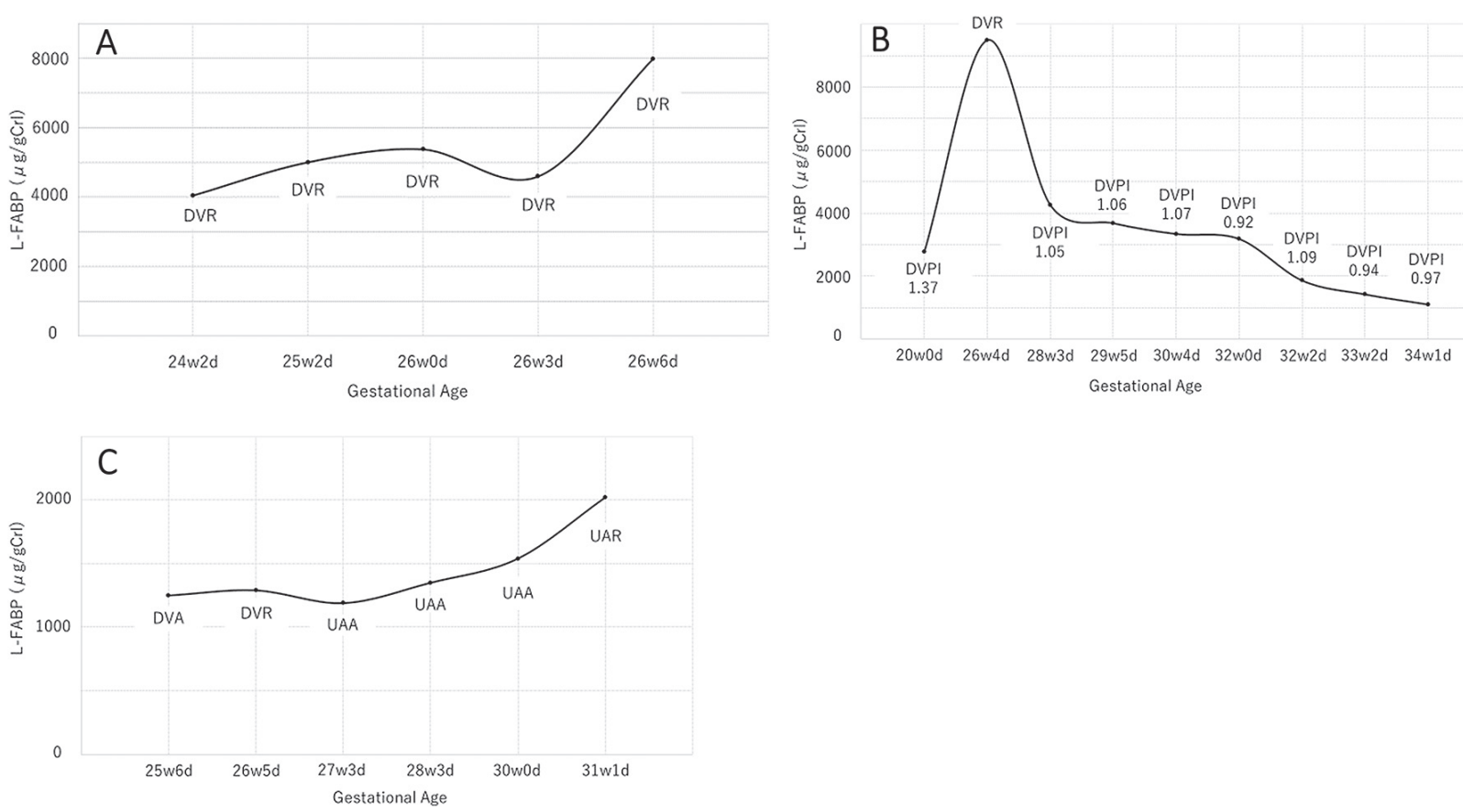

Fig. 1. Changes in the amniotic fluid level of liver type-fatty acid binding protein and fetal blood flow abnormalities during pregnancy: (A) Case 1, (B) Case 2, and (C) Case 3.

DVA, ductus venosus with absent A-wave; DVPI, ductus venosus pulsatility index; DVR, ductus venosus with reversed A-wave; UAA, umbilical artery with absent end-diastolic velocity; UAR, umbilical artery reversed end-diastolic velocity; w, weeks; d, days. 
samples. Ductus venosus with a reversed A-wave implies imminent fetal death (Baschat 2010). Therefore, elevated amniotic fluid L-FABP levels in cases with ductus venosus with a reversed A-wave is reasonable compared with other fetal blood flow abnormalities. Although we speculated that there could be a correlation between the deterioration of fetal blood flow abnormalities and an increase in amniotic fluid L-FABP levels, no correlation was observed between the amniotic fluid L-FABP level and the ductus venosus pulsatility index. Additionally, as in cases 1,2, and 3 , there might be differences in the L-FABP levels in similar fetal blood flow abnormalities. In case 3 with neonatal death, the amniotic fluid L-FABP level at delivery was $7,990 \mu \mathrm{g} / \mathrm{gCr}$, which was particularly high for a case of ductus venosus with a reversed A-wave. The L-FABP levels might express fetal condition more sensitively than fetal Doppler velocity. Fetal condition and the intrauterine environment must be comprehensively evaluated based on the degree and course of fetal blood flow, including the umbilical and middle cerebral arteries and ductus venosus, as well as on fetal heart rate monitoring results. However, the amniotic fluid L-FABP level alone reflects the fetal condition and might be able to predict fetal deterioration and the intrauterine environment.

Evaluation of fetal Doppler velocity and fetal heart rate monitoring is subjective, and fetal Doppler velocity might be affected by fetal movement, uterine contractions ( $\mathrm{Li}$ et al 2006), and measurement site (Khare et al. 2006). Meanwhile, the amniotic fluid L-FABP level is objective and accurate, except in cases of turbidity. However, the normal values and changes in amniotic fluid L-FABP levels during pregnancy remain unknown. Additionally, amniocentesis must be performed to measure the L-FABP levels. Although amniocentesis has very few complications (Kalogiannidis et al. 2011), it is more invasive than ultrasonography and fetal heart rate monitoring.

Furthermore, although the L-FABP level is considered to be a prognostic marker for acute and chronic kidney injury, no correlation was observed between the amniotic fluid L-FABP and neonatal creatinine level. The amniotic fluid L-FABP level might be a more sensitive marker for fetal hypoxia and gestational immaturity than renal dysfunction.

This study has several limitations. First, the number of cases with fetal blood flow abnormalities was relatively small. Second, the amniotic fluid L-FABP levels during pregnancy were not systematically examined. Further studies are needed to evaluate the relationship between the amniotic fluid L-FABP level and fetal and neonatal renal function, since we were unable to measure other biomarkers for renal damage such as $\beta 2$-microglobulin and cystatin C. Additionally, amniocentesis is an invasive procedure.

Further prospective studies are needed to clarify the clinical applicability of amniotic fluid L-FABP levels as a predictor of fetal condition and the intrauterine environment. Amniotic fluid L-FBAP levels might be a useful marker to determine the time of delivery with respect to fetal death, and short- and long-term prognoses.

In conclusion, amniotic fluid L-FABP level is an objective and accurate predictive marker of the fetal condition and might be able to predict fetal deterioration and the intrauterine environment.

\section{Acknowledgments}

We would like to thank Editage (https://www.editage. com) for performing English language editing.

\section{Author Contributions}

D.K. conceived and designed the study, collected data, performed the analysis, and wrote the manuscript. S.T., K.H., S.T., R.Z., T.H., F.K., and N.K. collected data. T.M. discussed the results and contributed to the final manuscript.

\section{Conflict of Interest}

The authors declare no conflict of interest.

\section{References}

Asada, T., Isshiki, R., Hayase, N., Sumida, M., Inokuchi, R., Noiri, E., Nangaku, M., Yahagi, N. \& Doi, K. (2016) Impact of clinical context on acute kidney injury biomarker performances: differences between neutrophil gelatinase-associated lipocalin and L-type fatty acid-binding protein. Sci. Rep., 6, 33077.

Baschat, A.A. (2010) Ductus venosus Doppler for fetal surveillance in high-risk pregnancies. Clin. Obstet. Gynecol., 53, 858-868.

Baschat, A.A. (2011) Neurodevelopment following fetal growth restriction and its relationship with antepartum parameters of placental dysfunction. Ultrasound Obstet. Gynecol., 37, 501-514.

Baschat, A.A., Viscardi, R.M., Hussey-Gardner, B., Hashmi, N. \& Harman, C. (2009) Infant neurodevelopment following fetal growth restriction: relationship with antepartum surveillance parameters. Ultrasound Obstet. Gynecol., 33, 44-50.

Bennaars-Eiden, A., Higgins, L., Hertzel, A.V., Kapphahn, R.J., Ferrington, D.A. \& Bernlohr, D.A. (2002) Covalent modification of epithelial fatty acid-binding protein by 4-hydroxynonenal in vitro and in vivo. Evidence for a role in antioxidant biology. J. Biol. Chem., 277, 50693-50702.

Derikx, J.P., Poeze, M., van Bijnen, A.A., Buurman, W.A. \& Heineman, E. (2007) Evidence for intestinal and liver epithelial cell injury in the early phase of sepsis. Shock, 28, 544-548.

Figueras, F. \& Gratacos, E. (2014) Update on the diagnosis and classification of fetal growth restriction and proposal of a stage-based management protocol. Fetal Diagn. Ther, 36, 86-98.

Gluckman, P.D., Hanson, M.A. \& Beedle, A.S. (2007) Early life events and their consequences for later disease: a life history and evolutionary perspective. Am. J. Hum. Biol., 19, 1-19.

Gluckman, P.D., Hanson, M.A., Spencer, H.G. \& Bateson, P. (2005) Environmental influences during development and their later consequences for health and disease: implications for the interpretation of empirical studies. Proc. Biol. Sci., 272, 671-677.

Iwagaki, S., Takahashi, Y., Chiaki, R., Asai, K., Matsui, M., Mori, T. \& Kawabata, I. (2019) Hypercoiled cord can cause a reversible abnormal Doppler in ductus venosus in cases of fetal growth restriction. J. Obstet. Gynaecol. Res., 44, 19221928. 
Kalogiannidis, I., Prapa, S., Dagklis, T., Karkanaki, A., Petousis, S., Prapas, Y. \& Prapas, N. (2011) Amniocentesis-related adverse outcomes according to placental location and risk factors for fetal loss after midtrimester amniocentesis. Clin. Exp. Obstet. Gynecol., 38, 239-242.

Kanda, Y. (2013) Investigation of the freely available easy-to-use software 'EZR' for medical statistics. Bone Marrow Transplant., 48, 452-458.

Katsura, D., Takahashi, Y., Iwagaki, S., Chiaki, R., Asai, K., Koike, M., Nagai, R., Yasumi, S. \& Furuhashi, M. (2019) Amnioinfusion for variable decelerations caused by umbilical cord compression without oligohydramnios but with the sandwich sign as an early marker of deterioration. J. Obstet. Gynaecol., 39, 49-53.

Khare, M., Paul, S. \& Konje, J.C. (2006) Variation in Doppler indices along the length of the cord from the intraabdominal to the placental insertion. Acta Obstet. Gynecol. Scand., 85, 922-928.

Lees, C.C., Marlow, N., van Wassenaer-Leemhuis, A., Arabin, B., Bilardo, C.M., Brezinka, C., Calvert, S., Derks, J.B., Diemert, A., Duvekot, J.J., Ferrazzi, E., Frusca, T., Ganzevoort, W., Hecher, K., Martinelli, P., et al. (2015) 2 year neurodevelopmental and intermediate perinatal outcomes in infants with very preterm fetal growth restriction (TRUFFLE): a randomised trial. Lancet, 385, 2162-2172.

Li, H., Gudmundsson, S. \& Olofsson, P. (2006) Acute centralization of blood flow in compromised human fetuses evoked by uterine contractions. Early Hum. Dev., 82, 747-752.

Manning, F.A. (1999) Fetal biophysical profile. Obstet. Gynecol. Clin. North Am., 26, 557-577.
Shinozuka, N. (2002) Fetal biometry and fetal weight estimation: JSUM standardization. Ultrasound Rev. Obstet. Gynecol., 2, 156-161.

Takahashi, Y., Iwagaki, S., Chiaki, R., Iwasa, T., Takenaka, M., Kawabata, I. \& Itoh, M. (2014) Amnioinfusion before 26 weeks' gestation for severe fetal growth restriction with oligohydramnios: preliminary pilot study. J. Obstet. Gynaecol. Res., 40, 677-685.

The GRIT Study Group (Growth Restriction Intervention Trial) (1996) When do obstetricians recommend delivery for a highrisk preterm growth-retarded fetus? Eur. J. Obstet. Gynecol. Reprod. Biol., 67, 121-126.

Tonomura, Y., Uehara, T., Yamamoto, E., Torii, M. \& Matsubara, M. (2011) Decrease in urinary creatinine in acute kidney injury influences diagnostic value of urinary biomarker-tocreatinine ratio in rats. Toxicology, 290, 241-248.

Vossbeck, S., de Camargo, O.K., Grab, D., Bode, H. \& Pohlandt, F. (2001) Neonatal and neurodevelopmental outcome in infants born before 30 weeks of gestation with absent or reversed enddiastolic flow velocities in the umbilical artery. Eur. J. Pediatr., 160, 128-134.

Wang, G., Gong, Y., Anderson, J., Sun, D., Minuk, G., Roberts, M.S. \& Burczynski, F.J. (2005) Antioxidative function of L-FABP in L-FABP stably transfected chang liver cells. Hepatology, 42, 871-879.

Yamamoto, T., Noiri, E., Ono, Y., Doi, K., Negishi, K., Kamijo, A., Kimura, K., Fujita, T., Kinukawa, T., Taniguchi, H., Nakamura, K., Goto, M., Shinozaki, N., Ohshima, S. \& Sugaya, T. (2007) Renal L-type fatty acid--binding protein in acute ischemic injury. J. Am. Soc. Nephrol., 18, 2894-2902. 